\title{
Partial deletion of the ROCK2 protein fails to reduce renal fibrosis in a unilateral ureteral obstruction model in mice
}

\author{
ITSUKO BABA, YASUHIRO EGI and KAZUO SUZUKI \\ Pharmacology Research Laboratories II, Research Division, Mitsubishi Tanabe Pharma Corporation, \\ Toda-Shi, Saitama 335-8505, Japan
}

Received December 5, 2014; Accepted September 29, 2015

DOI: $10.3892 / \mathrm{mmr} .2015 .4569$

\begin{abstract}
Renal fibrosis is a well-known cause for the progression of chronic kidney disease. Rho/Rho-associated coiled-coil kinase (ROCK) signaling is involved in renal fibrotic processes. Non-selective ROCK $1 / 2$ inhibitors have been reported to reduce renal interstitial fibrosis in a rodent unilateral ureteral obstruction (UUO) model. To clarify the role and contribution of ROCK2 in renal fibrosis, the present study used ROCK2 heterozygous knockout (HKO) mice to assess collagen deposition and fibrosis-associated gene expression in the kidney of the UUO model. In the ROCK2 HKO mice, the expression level of ROCK 2 in the normal kidney was half of that in the kidney of wild-type (WT) mice. The expression levels of ROCK1 in the ROCK2 HKO mice and WT mice were equivalent. Furthermore, in the ROCK2 HKO and the WT mice, the hydroxyproline content and the gene expression levels of collagen I and transforming growth factor- $\beta 1$ in the obstructed kidneys were augmented following UUO. By contrast, the mRNA expression of $\alpha$-smooth muscle actin decreased in the ROCK2 HKO mice, compared with that in the WT mice. The activity of ROCK in the obstructed kidneys, indicated by the phosphorylation of myosin phosphatase target subunit-1, which is a non-selective substrate of ROCK1 and ROCK2, was equivalent among the ROCK2 HKO and WT mice. In conclusion, no differences in renal interstitial fibrosis or UUO-induced ROCK activity were identified between the ROCK2 HKO and WT mice, indicating that the genetic partial disruption of ROCK2 is insufficient for protecting against renal fibrosis.
\end{abstract}

Correspondence to: Ms. Itsuko Baba, Pharmacology Research Laboratories II, Research Division, Mitsubishi Tanabe Pharma Corporation, 2-2-50 Kawagishi, Toda-Shi, Saitama 335-8505, Japan E-mail: baba.itsuko@mk.mt-pharma.co.jp

Abbreviations: ROCK, Rho-associated coiled-coil kinase; UUO, unilateral ureteral obstruction; HKO, heterozygous knockout

Key words: unilateral ureteral obstruction, renal interstitial fibrosis, Rho-associated coiled-coil kinase, heterozygous knockout mice, myofibroblast

\section{Introduction}

Rho-associated coiled-coil kinase (ROCK) is a serine/threonine kinase comprising two isoforms, ROCK1 and ROCK2. The small Rho G-protein activates ROCK, which phosphorylates several downstream targets $(1,2)$. The Rho/ROCK signaling pathway has been shown to contribute to a variety of cell functions, including smooth muscle contraction, stress fiber formation, cell proliferation and cell migration (3-5). The expression of ROCK1 is distributed in the kidneys, liver, lungs, spleen and testes, whereas ROCK2 is particularly expressed in the heart and brain $(1,2,6)$. The pharmacological inhibition of ROCK indicates that ROCK is important in cardiovascular diseases, including hypertension, heart failure and chronic kidney disease (7-14).

Several reports in the cardiovascular field have focused on the association between ROCK and tissue fibrosis. In a rat coronary artery occlusion model, the inhibition of ROCK by fasudil, a non-selective ROCK1/2 inhibitor, reduced the expression of inflammatory cytokines, including transforming growth factor- $\beta 1$ (TGF- $\beta 1$ ) and macrophage migration inhibitory factor, and prevented cardiomyocyte hypertrophy and interstitial fibrosis (15). Furthermore, fasudil attenuated the upregulation in the expression of profibrotic genes, including collagen I and III, in the heart following pressure overload in transverse aortic constriction (TAC) model mice, and ameliorated myocardial remodeling and fibrosis (16). In terms of kidney diseases, fasudil has been observed to inhibit the activation of ROCK and the TGF- $\beta$-small mothers against decapentaplegic (Smad) pathway, preventing glomerulosclerosis and tubulointerstitial fibrosis in an aldosterone-induced renal injury model (17). In a unilateral ureteral obstruction (UUO) model, the inhibition of ROCK by Y-27632 or fasudil inhibited the activity of ROCK and the expression of fibrosis-associated genes, including collagen, TGF- $\beta 1$, and $\alpha$-smooth muscle actin ( $\alpha$-SMA), thereby preventing tubulointerstitial fibrosis $(18,19)$. These findings suggest that ROCK is important in the development of tissue fibrosis.

Further investigations using ROCK1 or ROCK2 knockout (KO) mice have been reported. The deletion of ROCK1 has been demonstrated to suppress cardiac fibrosis and ventricular remodeling following pressure overload by TAC (20) and protects the development of albuminuria in a streptozotocin-induced diabetic kidney disease model (21). Following 
cardiac-specific deletion of ROCK2, a previous study reported that angiotensin II-induced cardiac hypertrophy and fibrosis were attenuated, compared with those in WT mice (22). These reports, as well as previous studies involving ROCK inhibitors, suggest that ROCK1 and ROCK2 contribute to cardiac inflammation and fibrosis in the development of heart failure. By contrast, the deletion of ROCK1 in a previous study did not affect the expression levels of $\alpha$-SMA, or collagen I and III within the diseased kidney of a UUO model, nor did it prevent UUO-induced renal fibrosis (23). Thus, the role and contribution of ROCK2 in renal fibrosis remains to be fully elucidated. The present study assessed whether ROCK 2 is involved in the development of renal fibrosis following UUO in ROCK2 HKO mice.

\section{Materials and methods}

Animals. This study was approved by the Experimental Animal Care and Use Committee of Mitsubishi Tanabe Pharma Corporation (Saitama, Japan). Male ROCK2 HKO (24) and WT mice were purchased from Charles River Japan (Charles River Laboratories International, Kanagawa, Japan) and were maintained at room temperature on a $12 \mathrm{~h}$ light/dark cycle, and were allowed access to standard laboratory chow (CRF-1; Oriental Yeast, Tokyo, Japan) and tap water ad libitum. The animals were housed at the Animal Care Facility of Mitsubishi Tanabe Pharma Corporation, in accordance with the relevant protocols.

Unilateral ureteral obstruction model. The mice were anesthetized with sevofrane (Maruishi Pharmaceutical Co., Ltd., Osaka, Japan) and subjected to a left flank incision. UUO was performed by complete ligation of the left ureter at the ureteropelvic junction with a 4-0 silk suture (Niccho Kogyo Co., Ltd., Tokyo, Japan). Sham-operated mice had their ureter exposed without ligation. All mice used for experiments were 8-10 weeks of age following a 1 week acclimation period (WT, $\mathrm{n}=25$; HKO, $\mathrm{n}=38$ ). The mice were sacrificed under anesthesia with sevofrane on days 7 and 14 following surgery. For RNA and hydroxyproline analyses, 42 mice [sham, WT $(n=5)$ and HKO $(n=8)$; day 7, WT $(n=5)$ and HKO $(n=9)$; day 14, WT $(n=5)$, HKO $(n=10)]$, and for protein analysis, 21 mice [sham, WT $(n=7)$ and HKO $(n=7)$; day 7 , WT $(n=3)$ and HKO $(n=4)]$ were sacrificed under anesthesia with sevofrane. The kidneys were then removed and divided into two sections for the subsequent analyses of RNA and hydroxyproline. For protein analysis, the whole kidneys were used.

Determination of kidney hydroxyproline content. The collagen content in the kidney was determined by hydroxyproline using a modified version of a previously described method $(25,26)$. In brief, the kidneys were homogenized in phosphate-buffered saline (Gibco; Thermo Fisher Scientific, Inc., Waltham, MA, USA), at $700 \mu \mathrm{l} / 100 \mathrm{mg}$ kidney weight, and were completely hydrolyzed in $6 \mathrm{~mol} / 1 \mathrm{HCl}$ (Wako Pure Chemical Industries, Ltd., Osaka, Japan) at $120^{\circ} \mathrm{C}$ for $6 \mathrm{~h}$, and filtered through a $0.45-\mu \mathrm{m}$ Millex-HV filter (Merck Millipore, Hessen, Germany). The samples were dried by vacuum centrifugation using an EZ-2 plus (Genevac, Ltd., Suffolk, UK) for $16 \mathrm{~h}$. The dried samples were solubilized in distilled water (2/3 volume of hydrolyzed sample). The samples were oxidized using chloramine T solution, containing $1.4 \%$ sodium $p$-toluenesulfonchloramide trihydrate (chloramine T; Wako Pure Chemical Industries, Ltd.) and 10\% n-propanol (Wako Pure Chemical Industries, Ltd.) in citric acid buffer, which consisted of $0.26 \mathrm{~mol} / 1$ citric acid (Sigma-Aldrich, St. Louis, MO, USA), $0.88 \mathrm{~mol} / \mathrm{l}$ sodium acetate trihydrate (Wako Pure Chemical Industries, Ltd.), $0.85 \mathrm{~mol} / 1$ sodium hydroxide (Wako Pure Chemical Industrie, Ltd.) and 1.2\% acetic acid (Kanto Chemical Co., Inc., Tokyo, Japan). Following incubation at room temperature for $20 \mathrm{~min}$, Ehrlich's solution comprising $1 \mathrm{~mol} / 1$ 4-dimethylaminobenzaldehyde (Sigma-Aldrich), $18 \%$ perchloric acid (Sigma-Aldrich) and 60\% $n$-propanol, was added, and the samples were incubated at $65^{\circ} \mathrm{C}$ for $40 \mathrm{~min}$. Subsequently, the absorbance was measured at $560 \mathrm{~nm}$ using a SpectraMax M5e microplate reader with SoftMax Pro ver. 5.4.1 (Molecular Devices; Thermo Fisher Scientific, Inc.). The concentration of hydroxyproline was estimated using a standard curve, using a pure solution of $l$-hydroxyproline (Wako Pure Chemical Industries, Inc.), with the final results expressed as hydroxyproline/mg protein. The kidney protein concentrations were determined using a BCA Protein Assay (Pierce; Thermo Fisher Scientific, Inc.) with bovine serum albumin as a standard.

\section{Reverse transcription-quantitative polymerase chain reaction} (RT-qPCR) analyses. The kidneys were homogenized and total RNA was extracted using TRIzol reagent (Invitrogen; Thermo Fisher Scientific, Inc.) and purified according to the manufacturer's protocol of an RNeasy Mini Kit (cat. no. 74106; Qiagen, Venlo, Netherlands). The total RNA concentration was determined using a NanoDrop 1000 Spectrophotometer (Thermo Fisher Scientific, Inc.). cDNA was synthesized from $1 \mu \mathrm{g}$ of total RNA using SuperScript VILO Master mix (cat. no. 11755250; Invitrogen; Thermo Fisher Scientific, Inc.) using an iCycler Thermal Cycler (Bio-Rad Laboratories, Inc., Hercules, CA, USA) under the following conditions: $25^{\circ} \mathrm{C}$ for $10 \mathrm{~min}, 42^{\circ} \mathrm{C}$ for $60 \mathrm{~min}$ and $85^{\circ} \mathrm{C}$ for $5 \mathrm{~min}$. qPCR was performed with $1 \mu \mathrm{l}$ cDNA in a total reaction volume of $20 \mu 1$ using a 7500 Fast Real-Time PCR system (Applied Biosystems; Thermo Fisher Scientific, Inc.) using TaqMan technology. The cycling conditions were as follows: $50^{\circ} \mathrm{C}$ for $2 \mathrm{~min}, 95^{\circ} \mathrm{C}$ for $10 \mathrm{~min}$ and 40 cycles of $95^{\circ} \mathrm{C}$ for $15 \mathrm{sec}$ and $60^{\circ} \mathrm{C}$ for $1 \mathrm{~min}$. Data were analyzed using the standard curve method. The results of each gene were normalized to that of 18SrRNA as an internal control. The following TaqMan Gene Expression

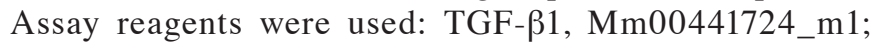
$\alpha$-SMA, Mm01546133_m1; collagen 1a2, Mm00483888_m1 and 18SrRNA, 4308329 (all from Applied Biosystems; Thermo Fisher Scientific, Inc.).

Western blot analysis. The kidney tissues were homogenized in lysis buffer containing $50 \mathrm{mmol} / 1$ Tris- $\mathrm{HCl}(\mathrm{pH} \mathrm{8.0}$; Nacalai Tesque, Inc., Kyoto, Japan), 150 mmol/1 NaCl (Wako Pure Chemical Industries, Inc.), $0.5 \%$ sodium deoxycholate (Wako Pure Chemical Industries, Inc.), $0.1 \%$ sodium dodecyl sulfate (Bio-Rad Laboratories, Inc.), 1\% Triton X-100 (Sigma-Aldrich), 1x protease inhibitors (complete; cat. no. 11697498001; Roche Diagnostics, Basel, Switzerland), and $1 \mathrm{x}$ phosphatase inhibitors (P2850; Sigma-Aldrich). 
Following centrifugation at $15,000 \mathrm{x}$ g for $20 \mathrm{~min}$ at $4{ }^{\circ} \mathrm{C}$ (CF-15R; Hitachi, Ltd., Tokyo, Japan), the supernatant was collected as lysate. The lysates were denatured for $5 \mathrm{~min}$ by boiling. Protein concentration was determined using a BCA Protein Assay (Pierce; Thermo Fisher Scientific, Inc.) with bovine serum albumin as a standard. Equal quantities of lysate were separated on a 4-15\% TGX Precast Gel (Bio-Rad Laboratories, Inc.) and transferred onto a polyvinylidene difluoride membrane (Bio-Rad Laboratories, Inc.) using the Trans-Blot Turbo Transfer System (Bio-Rad Laboratories, Inc.). The blots were blocked in Starting Block T20 (PBS) blocking buffer (Pierce; Thermo Fisher Scientific, Inc.) at room temperature for $1 \mathrm{~h}$. They were subsequently incubated overnight at $4^{\circ} \mathrm{C}$ with the following primary antibodies: Anti-human ROCK1 rabbit polyclonal antibody (1:200; H-85; cat. no. sc-5560, Santa Cruz Biotechnology, Inc., Santa Cruz, CA, USA), anti-chicken $\beta$-actin mouse monoclonal antibody (1:1,000; C4; cat. no. sc-47778; Santa Cruz Biotechnology, Inc.), anti-rat ROCK 2 mouse monoclonal antibody (1:5,000; cat. no. 610624; Transduction Laboratories; BD Biosciences, Franklin Lakes, NJ, USA), anti-human myosin phosphatase target subunit-1 (MYPT-1) rabbit polyclonal antibody (1:1,000; cat. no. 2634; Cell Signaling Technology Inc., Danvers, MA, USA) and anti-human phosphorylated (p)-MYPT-1 (Thr853) rabbit polyclonal antibody $(1: 1,000$; cat. no. 4563; Cell Signaling Technology, Inc.) in Can Get Signal solution (Toyobo Co., Ltd., Osaka, Japan). The membranes were then incubated with the following secondary polyclonal antibodies: ECL donkey anti-rabbit IgG horseradish peroxidase (HRP)-conjugated species-specific whole antibody (1:5,000; cat. no. NA934; GE Healthcare Life Sciences, Buckinghamshire, UK) and ECL sheep anti-mouse IgG, HRP-conjugated species-specific whole antibody (1:5,000; cat. no. NA931; GE Healthcare Life Sciences) at room temperature for $1 \mathrm{~h}$. The membranes were developed using enhanced chemiluminescence methods (LumiGLO Reserve Chemiluminescent Substrate kit; cat. no. 54-71-00; KPL, Gaithersburg, MD, USA). The signal intensities of the specific bands were detected using an LAS-3000 Luminescent Image Analyzer (Fujifilm, Tokyo, Japan) and analyzed with Multi Gauge (ver. 3.0; Fujifilm). For quantification, the signal intensities were normalized to $\beta$-actin loaded in each well.

Statistical analyses. All data were expressed as the mean \pm standard error of the mean. Parameters between the sham- and UUO-operated mice, or between the WT and ROCK 2 HKO mice were compared using Student's $t$-test. Statistical analyses were performed using SAS system, version 8.0.0 (SAS Institute, Cary, NC, USA) in the biometrics section. $\mathrm{P}<0.05$ was considered to indicate a statistically significant difference.

\section{Results}

Hydroxyproline content in UUO kidneys. To assess renal interstitial fibrosis, the present study evaluated the accumulation of collagen in the UUO kidneys. Total kidney collagen deposition was determined by measuring the hydroxyproline content, which significantly increased in the UUO-operated kidney, compared with that of the sham-operated kidney.

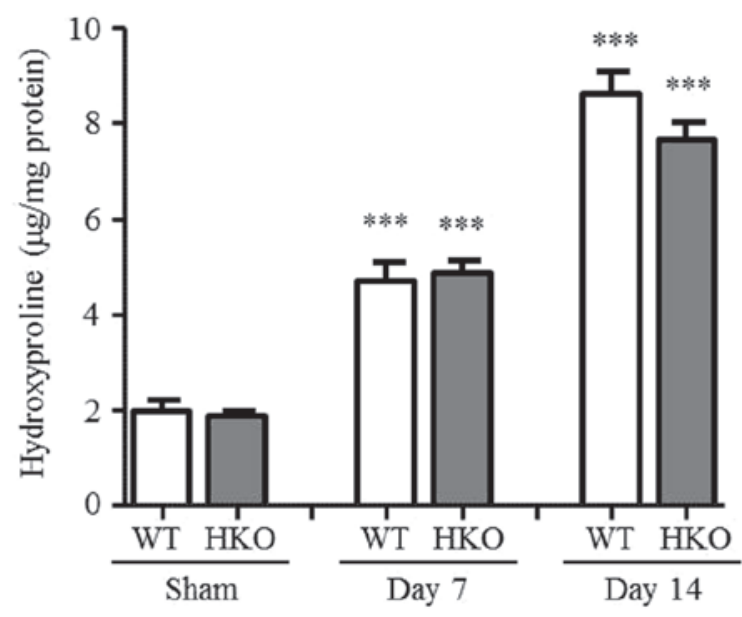

Figure 1. Hydroxyproline content in the kidney tissues of WT and HKO mice at days 7 and 14 following UUO. The hydroxyproline contents in the kidney tissues of the UUO-operated mice were compared with those of the sham-operated mice 7 and 14 days following UUO. Results are expressed as mean \pm standard error of the mean $(n=5-10){ }^{* * * *} \mathrm{P}<0.001$, compared with the sham group. WT, wild-type; HKO, heterozygous knockout of Rho/Rho-associated coiled-coil kinase 2; UUO, unilateral ureteral obstruction.

Following 3 days of UUO, the hydroxyproline content continued to increase (data not shown). On days 7 and 14 post-UUO, the WT and HKO mice were compared. Compared with the sham-operated group, the hydroxyproline content increased in the UUO-operated kidney of the WT mice (2.4and 4.4-fold on days 7 and 14 post-UUO, respectively) and HKO mice (2.6- and 4.1-fold at days 7 and 14 post-UUO, respectively), as shown in Fig. 1. No significant differences were identified.

mRNA expression levels of collagen I, $\alpha$-SMA and TGF- $\beta 1$ in UUO kidneys. The present study measured the expression levels of fibrosis-associated genes to examine the effect of partial ROCK2 deletion. As shown in Fig. 2A-C, the mRNA expression levels of collagen I, $\alpha$-SMA and TGF- $\beta 1$ in the UUO-operated kidneys of the WT mice markedly increased, compared with those in the sham-operated mice (collagen I, 11.0- and 11.8-fold; $\alpha$-SMA, 5.1- and 6.9-fold; TGF- $\beta 1,7.9$ and 7.9-fold at days 7 and 14 post-UUO, respectively). By contrast, the mRNA expression level of $\alpha$-SMA was markedly ameliorated in the HKO mice at day 7 post-UUO, and was $78 \%$ of the level measured in the WT mice (Fig. 2B). At day 14 post-UUO, no statistically significant difference was observe in the mRNA expression level of $\alpha$-SMA in the HKO mice; however, the level of expression was suppressed to $81 \%$ of that in the WT mice. In addition, the mRNA expression levels of collagen I and TGF- $\beta 1$ increased in the UUO-operated kidneys of the HKO mice (collagen I, 9.9- and 10.8-fold; TGF- $\beta 1,6.9$ and 7.4-fold at days 7 and 14 post-UUO, respectively); and their expression levels were almost the same as those observed in the WT mice (Fig. 2A and C).

ROCK activity in UUO kidneys. To confirm the difference in ROCK activity between the WT and HKO mice, the present study measured the phosphorylation of MYPT-1, a target protein of ROCK in the UUO-operated kidney. The phosphorylation 
A

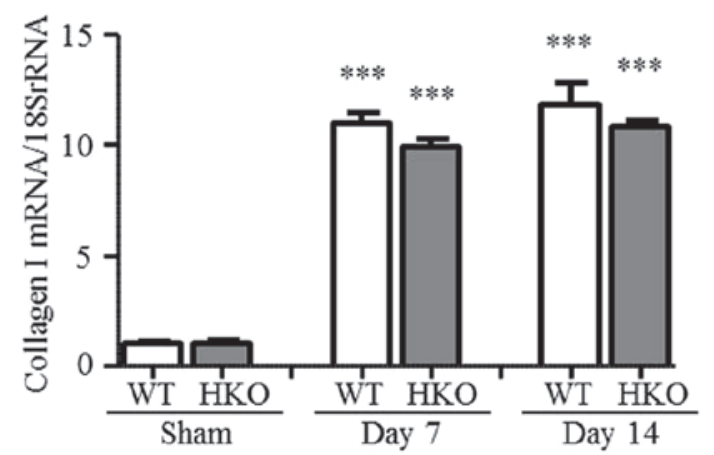

B

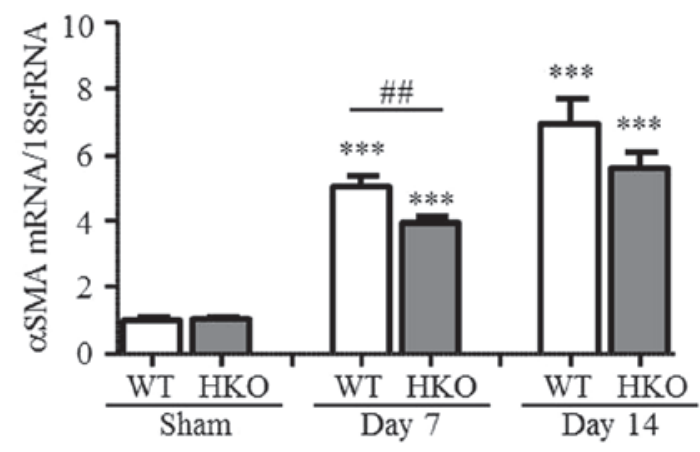

C

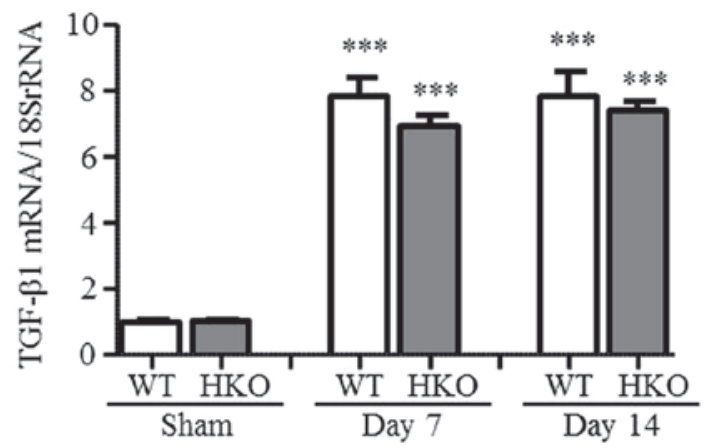

Figure 2. mRNA expression levels of collagen I, $\alpha$-SMA and TGF- $\beta 1$ in the kidney tissues of WT and HKO mice on days 7 and 14 following UUO. The mRNA expression levels of (A) collagen I, (B) $\alpha$-SMA and (C) TGF- $\beta 1$ in the UUO-operated mice were compared with those in the sham-operated mice on days 7 and 14 following UUO. The results are expressed as mean \pm standard error of the mean $(\mathrm{n}=5-10) .{ }^{* * *} \mathrm{P}<0.001$, compared with the sham group; ${ }^{\# \#} \mathrm{P}<0.01$, compared with the WT mice at the same time point. WT, wild-type; $\mathrm{HKO}$, heterozygous knockout of Rho/Rho-associated coiled-coil kinase 2 $\alpha$-SMA, $\alpha$-smooth muscle actin; TGF- $\beta 1$, transforming growth factor- $\beta 1$; UUO, unilateral ureteral obstruction.

of MYPT-1 was detected in the UUO-operated kidney of the WT and HKO mice in a similar manner (Fig. 3A and B), indicating that the activity of ROCK was not altered following the partial deletion of ROCK2. In the normal kidneys, the protein expression level of ROCK2 in the HKO mice was $50 \%$ of that observed in the WT mice, however, no differences were observed in the protein expression level of ROCK1 between the WT and HKO mice kidneys (Fig. 4A and B). The expression ratio of ROCK1 and ROCK2 was also unchanged in the UUO-operated kidney (data not shown).
A

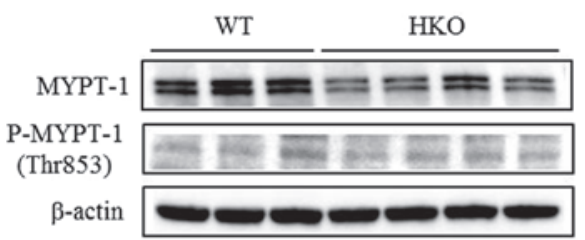

B

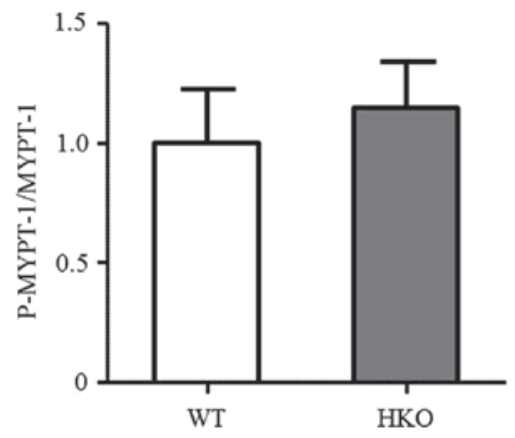

Figure 3. Activity of Rho/Rho-associated coiled-coil kinase in the kidney tissues of WT and HKO mice (A) Representative western blot indicate the protein levels of MYPT-1 (upper panel) and p-MYPT-1 (middle panel) proteins in the kidney tissues of the WT and HKO mice 7 days following UUO. The same blot was detected with $\beta$-actin (lower panel) to confirm equal loading. (B) Quantitative analysis shows the relative abundance of p-MYPT-1 and MYPT-1, as the p-MYPT-1/MYPT-1 ratio, following normalization with $\beta$-actin. Results are expressed as the mean \pm standard error of the mean $(n=3-4)$. WT, wild-type; HKO, heterozygous knockout of Rho/Rho-associated coiled-coil kinase 2; UUO, unilateral ureteral obstruction; MYPT-1, myosin phosphatase target subunit-1; p-, phosphorylated.

A

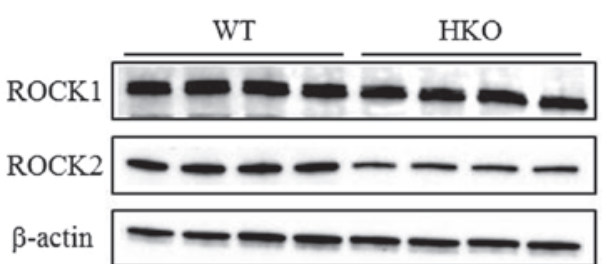

B

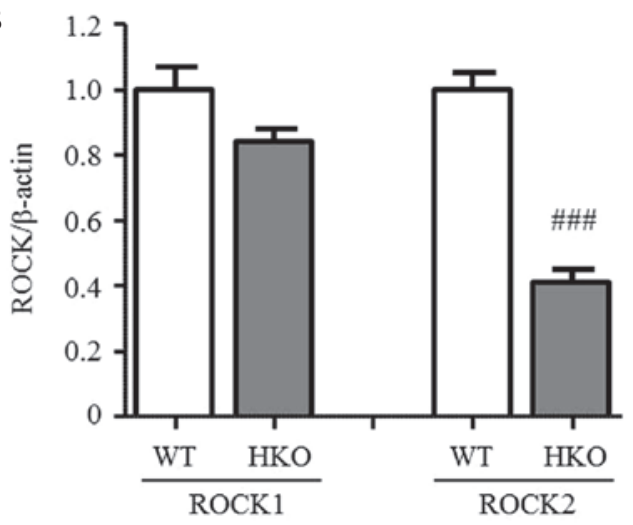

Figure 4. Protein expression levels of ROCK1 and ROCK2 in normal kidneys of WT and HKO mice. (A) Representative western blots show the protein levels of ROCK1 (upper panel) and ROCK2 (middle panel) in the normal kidneys of WT and HKO mice. $\beta$-actin (lower panel) was used to confirm equal loading. (B) Quantitative analysis of the relative abundance of the ROCK1 and ROCK 2 protein following normalization with $\beta$-actin. Results are expressed as the mean \pm standard error of the mean $(n=7) .{ }^{\# \#} \mathrm{P}<0.001$, compared with the WT mice. ROCK, Rho/Rho-associated coiled-coil kinase; WT, wild-type; HKO, heterozygous knockout of ROCK2; UUO, unilateral ureteral obstruction. 


\section{Discussion}

In the present study, the effects of the partial deletion of ROCK2 protein on the expression of fibrosis-associated genes and renal interstitial fibrosis were investigated in a UUO model in mice. In the WT mice, the hydroxyproline content and mRNA expression of collagen I in the UUO-operated kidneys were significantly increased at days 7 and 14 following UUO. The fibrotic parameters in the UUO kidneys of the ROCK2 HKO mice also increased. However, no statistical differences were identified between the WT and ROCK2 HKO mice on the increment of these fibrotic parameters in the UUO-operated kidneys, suggesting that the partial deletion of the ROCK2 protein does not affect the level of renal interstitial fibrosis induced by UUO. It has been reported in normal mice that Y27632, a non-selective ROCK1/2 inhibitor, suppresses the mRNA expression of collagen I and the deposition of collagen in damaged kidneys 4 and 10 days following UUO (18). In addition, in rat UUO model, fasudil was found to ameliorate collagen deposition in the damaged kidney 16 days following UUO (19). These results suggest that the pharmacological inhibition of ROCK can prevent the development of UUO-induced renal interstitial fibrosis. By contrast, other studies have demonstrated that, in ROCK1 KO mice, complete ROCK1 deletion did not affect collagen accumulation or the mRNA expression levels of collagen I in the UUO kidney 5 and 10 days following UUO. This suggests that ROCK2, but not ROCK1, is involved in renal interstitial fibrosis in UUO (23). However, in the present study, partial ROCK 2 deletion indicated a non-significant reduction in renal interstitial fibrosis. Thus, it is likely that the effect of the pharmacological inhibition of ROCK is inconsistent with that of the deletion of ROCK protein on the development of renal interstitial fibrosis following UUO.

It is evident that TGF- $\beta 1$ signals and the Rho/ROCK pathway are closely associated with renal interstitial fibrosis by UUO (27). Y27632 is reported to suppress the augmentation of the mRNA expression of TGF- $\beta 1$ in UUO mice (18). However, in ROCK1 KO mice, the expression of TGF- $\beta 1$ was found to be significantly enhanced in the damaged kidney following UUO, compared with WT mice, indicating that the absence of ROCK1 may not be able to suppress the expression of TGF- $\beta 1$ to protect against renal fibrosis (23). Therefore, ROCK2 was considered to be important in TGF- $\beta 1$ signaling in renal interstitial fibrosis following UUO. In the present study, partial ROCK2 deletion did not suppress the mRNA expression of TGF- $\beta 1$ or ROCK1, and ROCK activity remained unaffected in the UUO-operated kidney. These results suggested that the response to partial ROCK2 deletion was distinct from that to pharmacological ROCK inhibition.

In renal interstitial fibrosis, the transformation to myofibroblasts, which are considered to be the dominant collagen producing cells, is a crucial step toward collagen synthesis and deposition. In kidney fibrosis, the resident fibroblast, epithelial, endothelial and bone marrow-derived cells can acquire the phenotype of myofibroblasts and express the characteristic proteins, including $\alpha$-SMA (27-29). Furthermore, the ROCK signaling pathway is reported to be important in the transformation of cells into activated myofibroblasts $(30,31)$. In the present study, the expression level of $\alpha$-SMA was markedly decreased in the UUO kidney tissues of the ROCK2 HKO mice, compared with that of the WT mice. In the ROCK1 KO mice, the augmentation of mRNA and protein expression levels of $\alpha$-SMA caused by UUO were not decreased (23). Thus, it is likely that ROCK2 may be implicated in UUO-induced transformation via a signal cascade independent of TGF- $\beta 1$. In addition, the present study found in the mice UUO model, that the effect of ROCK2 HKO was inconsistent with the effect of the pharmacological inhibition of ROCK. With respect to this inconsistency, ROCK inhibitors are considered to be poor in isoform selectivity and other target specificity. Y-27632 and fasudil equivalently inhibit ROCK1 and ROCK2 activity; and fasudil also inhibits protein kinase (PK)N, PKC, myosin light chain kinase and mitogen-activated protein kinase kinase 1 (32). Therefore, it can was suggested that the partial deletion of ROCK2 may be insufficient in suppressing UUO-induced fibrotic responses. In addition, it is possible that the results depend on the experimental procedures and pathophysiological conditions in the UUO model. In order to address these problems, further investigations are required using kidney-specific ROCK1 or ROCK2 deletion, and using more specific inhibitors for ROCK1 or ROCK2. In conclusion, using ROCK2 HKO mice, the present study demonstrated that the partial deletion of ROCK2, as with ROCK1 inhibition, is insufficient for effectively preventing renal interstitial fibrosis.

\section{Acknowledgements}

The authors would like to thank Dr Shuh Narumiya (Medical Innovation Center, Graduate School of Medicine, Kyoto University, Kyoto, Japan) for their permission to use the ROCK2 HKO mice. The authors would also like to thank Dr Akiyoshi Fukamizu and Dr Junji Ishida (Life Science Center, Tsukuba Advanced Research Alliance, Tsukuba University, Ibaraki, Japan) for their guidance and support, and would like to thank Dr Kenji Arakawa, Dr Rikako Yamauchi and Dr Taku Sato (Mitsubishi Tanabe Pharma Corporation) for their support.

\section{References}

1. Schofield AV and Bernard O: Rho-associated coiled-coil kinase (ROCK) signaling and disease. Crit Rev Biochem Mol Biol 48: 301-316, 2013.

2. Amin E, Dubey BN, Zhang SC, Gremer L, Dvorsky R, Moll JM, Taha MS, Nagel-Steger L, Piekorz RP, Somlyo AV and Ahmadian MR: Rho-kinase: Regulation, (dys) function and inhibition. Biol Chem 394: 1399-1410, 2013.

3. Satoh K, Fukumoto Y and Shimokawa H: Rho-kinase: Important new therapeutic target in cardio-vascular diseases. Am J Physiol Heart Circ Physiol 301: H287-H296, 2011.

4. Hahmann C and Schroeter T: Rho-kinase inhibitors as therapeutics: From pan inhibition to isoform selectivity. Cell Mol Life Sci 67: 171-177, 2010.

5. Olson MF: Applications for ROCK kinase inhibition. Curr Opin Cell Biol 20: 242-248, 2008.

6. Shi J and Wei L: Rho kinase in the regulation of cell death and survival. Arch Immunol Ther Exp (Warsz) 55: 61-75, 2007.

7. Ming D, Yan BP, Liao JK, Lam YY, Yip GW and Yu CM: Rho-kinase inhibition: A novel therapeutic target for the treatment of cardiovascular diseases. Drug Discov Today 15: 622-629, 2010

8. Budzyn K, Marley PD and Sobey CG: Targeting Rho and Rho-kinase in the treatment of cardiovascular disease. Trends Pharmacol Sci 27: 97-104, 2006.

9. Shimokawa H and Rashid M: Development of Rho-kinase inhibitors for cardiovascular medicine. Trends Pharmacol Sci 28: 296-302, 2007. 
10. Kushiyama T, Oda T, Yamamoto K, Higashi K, Watanabe A Takechi H, Uchida T, Oshima N, Sakurai Y, Miura S and Kumagai H: Protective effects of Rho kinase inhibitor fasudil on rats with chronic kidney disease. Am J Physiol Renal Physiol 304: F1325-F1334, 2013.

11. Nishikimi T, Koshikawa S, Ishikawa Y, Akimoto K, Inaba C, Ishimura K, Ono H and Matsuoka H: Inhibition of Rho-kinase attenuates nephrosclerosis and improves survival in salt-loaded spontaneously hypertensive stroke-prone rats. J Hypertens 25: 1053-1063, 2007.

12. Kanda T, Wakino S, Hayashi K, Homma K, Ozawa Y and Saruta T: Effect of fasudil on Rho-kinase andv nephropathy in subtotally nephrectomized spontaneously hypertensive rats. Kidney Int 64: 2009-2019, 2003.

13. Xie X, Peng J, Chang X, Huang K, Huang J, Wang S, Shen X, Liu $\mathrm{P}$ and Huang $\mathrm{H}$ : Activation of RhoA/ROCK regulates NF- $\mathrm{KB}$ signaling pathway in experimental diabetic nephropathy. Mol Cell Endocrinol 369: 86-97, 2013.

14. Zhou H, Li YJ, Wang M, Zhang LH, Guo BY, Zhao ZS, Meng FL, Deng YG and Wang RY: Involvement of RhoA/ROCK in myocardial fibrosis in a rat model of type 2 diabetes. Acta Pharmacol Sin 32: 999-1008, 2011.

15. Rikitake Y, Oyama N, Wang CY, Noma K, Satoh M, Kim HH and Liao JK: Decreased perivascular fibrosis but not cardiac hypertrophy in ROCK1+/-haploinsufficient mice. Circulation 112 2959-2965, 2005.

16. Li Q, Xu Y, Li X, Guo Y and Liu G: Inhibition of Rho-kinase ameliorates myocardial remodeling and fibrosis in pressure overload and myocardial infarction: Role of TGF- $\beta 1$-TAK1 Toxicol Lett 211: 91-97, 2012.

17. Sun GP, Kohno M, Guo P, Nagai Y, Miyata K, Fan YY, Kimura S, Kiyomoto H, Ohmori K, Li DT, et al: Involvements of Rho-kinase and TGF-beta pathways in aldosterone-induced renal injury. J Am Soc Nephrol 17: 2193-2201, 2006.

18. Nagatoya K, Moriyama T, Kawada N, Takeji M, Oseto S, Murozono T, Ando A, Imai E and Hori M: Y-27632 prevents tubulointerstitial fibrosis in mouse kidneys with unilateral ureteral obstruction. Kidney Int 61: 1684-1695, 2002.

19. Satoh S, Yamaguchi T, Hitomi A, Sato N, Shiraiwa K, Ikegaki I Asano T and Shimokawa H: Fasudil attenuates interstitial fibrosis in rat kidneys with unilateral ureteral obstruction. Eur J Pharmacol 455: 169-174, 2002.

20. Zhang YM, Bo J, Taffet GE, Chang J, Shi J, Reddy AK, Michael LH, Schneider MD, Entman ML, Schwartz RJ and Wei L: Targeted deletion of ROCK1 protects the heart against pressure overload by inhibiting reactive fibrosis. FASEB J 20: 916-925, 2006.
21. Zhou L, Liu F, Huang XR, Chen H, Chung AC, Shi J, Wei L, Lan HY and Fu P: Amelioration of albuminuria in ROCK1 knockout mice with streptozotocin-induced diabetic kidney disease. Am J Nephrol 34: 468-475, 2011.

22. Okamoto R, Li Y, Noma K, Hiroi Y, Liu PY, Taniguchi M, Ito M and Liao JK: FHL2 prevents cardiac hypertrophy in mice with cardiac-specific deletion of ROCK2. FASEB J 27: 1439-1449, 2013.

23. Fu P, Liu F, Su S, Wang W, Huang XR, Entman ML, Schwartz RJ, Wei L and Lan HY: Signaling mechanism of renal fibrosis in unilateral ureteral obstructive kidney disease in ROCK1 knockout mice. J Am Soc Nephrol 17: 3105-3114, 2006.

24. Thumkeo D, Keel J, Ishizaki T, Hirose M, Nonomura K, Oshima H, Oshima M, Taketo MM and Narumiya S: Targeted disruption of the mouse Rho-associated kinase 2 gene results in intrauterine growth retardation and fetal death. Mol Cell Biol 23: 5043-5055, 2003.

25. Woessner JF: The determination of hydroxyproline in tissue and protein samples containing small proportions of this imino acid. Arch Biochem Biophys 93: 440-447, 1961.

26. Kivirikko KI, Laitinen O and Prockop DJ: Modifications of a specific assay for hydroxyproline in urine. Anal Biochem 19: 249-255, 1967.

27. Liu Y: Epithelial to mesenchymal transition in renal fibrogenesis: Pathologic significance, molecular mechanism and therapeutic intervention. J Am Soc Nephrol 15: 1-12, 2004.

28. Piera-Velazquez S, Li Z and Jimenez SA: Role of endothelial-mesenchymal transition (EndoMT) in the pathogenesis of fibrotic disorders. Am J Pathol 179: 1074-1080, 2011.

29. Duffield JS: Cellular and molecular mechanisms in kidney fibrosis. J Clin Invest 124: 2299-2306, 2014.

30. Patel S, Takagi KI, Suzuki J, Imaizumi A, Kimura T, Mason RM, Kamimura T and Zhang Z: RhoGTPase activation is a key step in renal epithelial mesenchymal transdifferentiation. J Am Soc Nephrol 16: 1977-1984, 2005.

31. Rodrigues-Díez R, Carvajal-González G, Sánchez-López E, Rodríguez-Vita J, Rodrigues Díez R, Selgas R, Ortiz A, Egido J, Mezzano S and Ruiz-Ortega M: Pharmacological modulation of epithelial mesenchymal transition caused by angiotensin II. Role of ROCK and MAPK pathways. Pharm Res 25: 2447-2461, 2008.

32. Tamura M, Nakao H, Yoshizaki H, Shiratsuchi M, Shigyo H, Yamada H, Ozawa T, Totsuka J and Hidaka H: Development of specific Rho-kinase inhibitors and their clinical application. Biochim Biovphys Acta 1754: 245-252, 2005. 